\title{
Source Reconstruction Technique for Slot Array Antennas using the Gerchberg-Papoulis Algorithm
}

\author{
Makoto Sano, Manuel Sierra-Castañer, Tamara Salmerón-Ruiz, Jiro Hirokawa, Makoto Ando
}

\begin{abstract}
A source reconstruction technique for slot array antennas is presented. By exploiting the information about the positions and the polarizations of slots to the Gerchberg-Papoulis iterative algorithm, the field on the slots is accurately reconstructed. The proposed technique is applied to the source reconstruction of a K-band radial line slot antenna (RLSA), and the simulated and measured results are presented.
\end{abstract}

Index Terms - antenna measurements, source reconstrucion, truncation error.

\section{INTRODUCTION}

One of the important parameters in antenna diagnosis is the field distribution on the antenna aperture. There are several methods to obtain the aperture field distribution. One of them is the application of the integral equation methods to the nearfield measurement [1], which is commercially available in in INSIGHT $^{\circledR}$ (Microwave Vision Group) or DIATOOL ${ }^{\circledR}$ (TICRA). This technique allows accurate reconstruction of the aperture field, however, it is very time-consuming.

Another technique is the application of the Fourier transform to the far field or to the measured planar near field [2], using the plane wave spectrum (PWS) [3]. In the case of cylindrical or spherical near-field measurement, this technique can be applied after performing the near-to-far field transformation [4]. This technique is time efficient because the use of the fast Fourier transform (FFT) allows very fast computation. However, the resolution is lower than half wavelength since only the visible range of the spectrum is used. Also, in the case of planar near field measurements, since the scan area is finite, so-called truncation error is caused [5]. In truncated measurements, only a certain portion called reliable region of the PWS is reliable. The reliable region corresponds to the coverage area of the radiated field from an antenna under test [6]. In the case of spherical near-field measurement, the PWS can also be obtained by transforming the spherical modes [7]. In this case also, the invisible region of the PWS is often disregarded because the measured spherical modes do not converge in the invisible region.

The Gerchberg-Papoulis iterative algorithm [8] has been applied to planar near-field measurements [9] in order to reduce the truncation error in antenna measurements. By applying filtering in both spatial and spectral domains, the PWS outside the reliable region is reconstructed. For the spatial filtering, only the information of the shape of the antenna is used, and field outside the physical extent of the antenna is truncated in the iterative procedure.

For a slot array antenna, much more information about the antenna such as positions and polarizations of the slots can be exploited. In this paper, the DFT is applied to the GerchbergPapoulis algorithm instead of the FFT in order to compute the PWS from the field on the slots. Besides, using the information of the positions and the polarizations of the slots, the accuracy and convergence of the source reconstruction are improved. The proposed algorithm can be used not only for the extrapolation of the radiation pattern but also for source reconstruction. One of the advantages of the proposed algorithm is that it does not require any modifications of the usual measurements. Another advantage is that the magnetic currents (in the case of slots) can be directly reconstructed, becoming an excellent tool for antenna designers.

In this paper, the source reconstruction technique for slot array antennas and the examples of the source reconstruction from simulated and measured data are presented.

\section{COMPuTATION OF THE Plane WAVE SPECTRUM FROM THE ELECTRIC FIELD ON THE SLOTS}

The PWS is defined as the Fourier transform of the tangential field on the antenna aperture $(z=0)$.

$$
A\left(k_{x}, k_{y}\right)=\int_{-\infty}^{\infty} \int_{-\infty}^{\infty} E(x, y) e^{-j\left(k_{x} x+k_{y} y\right)} d x d y
$$

where, $A\left(k_{x}, k_{y}\right)$ and $E(x, y)$ are the electric PWS and the electric field on the antenna aperture, respectively. Since the source of a slot is a magnetic current which is calculated from the aperture electric field, magnetic PWS and magnetic field are not considered. For the computation of the PWS, the FFT is usually implemented because of its great efficiency. In this case, the both the electric field and the PWS have to be tabulated on equally spaced rectangular grids, and the number of sampling points for the electric field has to be the same as that of the PWS.

In order to compute the PWS of a slot array with arbitrary positions and number of slots, the DFT is employed instead of the FFT. The PWS is directly computed from the electric field on the slots, without interpolation.

$$
A\left(k_{x}(m), k_{y}(m)\right)=\sum_{n=1}^{N} E(x(n), y(n)) e^{-j\left(k_{x}(m) x(n)+k_{y}(m) y(n)\right)}
$$


where the $(x(n), y(n))$ is the center position of the $n$-th slot, and the $N$ is the number of the slots. The DFT is implemented using the following matrix form.

$$
\mathbf{A}=\mathbf{W E}
$$

where,

$$
\begin{gathered}
\mathbf{A}=\left[A\left(k_{x}(1), k_{y}(1)\right), \ldots, A\left(k_{x}(M), k_{y}(M)\right)\right]^{T} \\
\mathbf{E}=[E(x(1), y(1)), \ldots, E(x(N), y(N))]^{T} \\
\mathbf{W}=\left[\begin{array}{ccc}
e^{-j\left(k_{x}(1) x(1)+k_{y}(1) y(1)\right)} & \cdots & e^{-j\left(k_{x}(1) x(N)+k_{y}(1) y(N)\right)} \\
\vdots & \ddots & \vdots \\
e^{-j\left(k_{x}(M) x(1)+k_{y}(M) y(1)\right)} & \cdots & e^{-j\left(k_{x}(M) x(N)+k_{y}(M) y(N)\right)}
\end{array}\right]
\end{gathered}
$$

In the most cases, the number of the slots $N$ and the number of the sampling points for PWS $M$ are not the same and the matrix $\mathbf{W}$ is a rectangular matrix whose inverse matrix does not exist. Hence, the inverse of the DFT is implemented using the pseudoinverse matrix $\mathbf{W}^{\dagger}[10]$.

$$
\mathbf{E}=\mathbf{W}^{\dagger} \mathbf{A}
$$

Fig. 1 shows the geometries for the planar and spherical near-field measurements. Since the acquisition plane is finite, only a certain portion of the PWS called reliable region which corresponds to the coverage area of the radiated field of the AUT is reliable [6]. The reliable regions of the PWS's are shown in Fig. 2. The reliable region of the PWS for planar near-field measurement is the intersection of two ellipses. In the spherical case, the reliable region is circle with the radius of $k_{0} \sin \theta$. Even if the acquisition plane is the whole hemisphere $\left(\theta=90^{\circ}\right)$, the reliable region is identical with the visible region and the invisible region is still unreliable. For the far-field pattern computation, only the visible region is sufficient, however, for source reconstruction, the invisible region of the PWS is required.

The required extent of the PWS for source reconstruction of a slot array antenna is obtained from the resolution which corresponds to the minimum slot separation of the antenna. Since the proposed algorithm is based on the discrete Fourier transform, the relationship between the extent of the PWS $\pm k_{x \max }$ and the slot separation $\Delta x$ is given by

$$
k_{x \max }=\frac{\pi}{\Delta x}
$$

In the case of the RLSA used for the source reconstruction in this paper, the minimum slot separation is $0.25 \lambda_{0}$ and thus the required extent of the PWS is a circle with the radius of $2 k_{0}$.

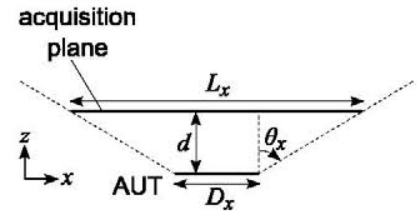

(a) planar near-field measurement

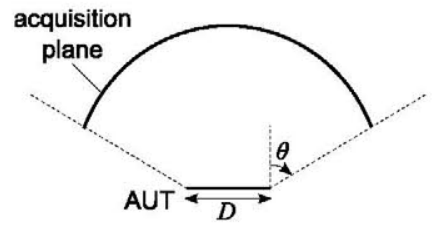

(b) spherical near-field measurement
Fig. 1. Geometries for the measurement setups of planar and spherical nearfield measurement. The $D$ denotes the dimension of the antenna under test (AUT). The geometry of the spherical near-field measurement setup is same as that of far-field measurement.

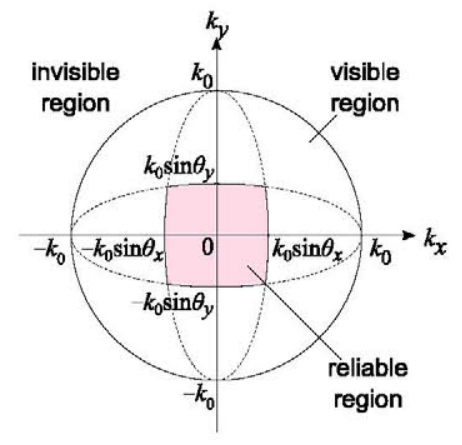

(a) planar near-field measurement

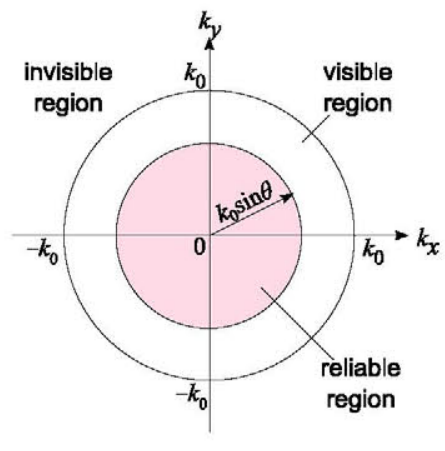

(b) spherical near-field measurement
Fig. 2. Reliable regions of the PWS's for the measurement setups shown in Fig. 1.

\section{ALGORITHM OF SOURCE RECONSTRUCTION}

The procedure of the source reconstruction is illustrated in Fig. 3. The major differences between the proposed algorithm and the algorithm [9] are the computation of the PWS and the spatial filtering applied during the iterative procedure. The computation of the PWS and the iterative part are briefly described in the following subsections

\section{A. Computation of the Initial Plane Wave Spectrum}

The initial PWS for the source reconstruction is obtained from planar, cylindrical, or spherical near-field measurement, as shown in the upper part of Fig. 1. In the case of the planar near-field measurement, the PWS is obtained by applying the fast Fourier transform. The probe correction is performed in order to eliminate the effect of the probe. In the case of cylindrical and spherical near-field measurements, at first the near-to-far field transformation is performed, and then the PWS is obtained by multiplying $(\cos \theta)^{-1}$ to the far field. The extent of the PWS is extended to have the spatial resolution corresponding to the minimum slot separation according to (8). Zero-padding is applied to the extended portion of the PWS.

\section{B. Iterative Procedure for Source Reconstruction}

After the initial PWS is obtained, the PWS outside the reliable region is extrapolated in the following steps.

Step 1) Compute the field on the slots by applying the inverse DFT using (7).

Step 2) Filter out the cross polar components of the slots. When the slot width is small with respect to the slot length, the 
magnetic current is almost along the slot, and the cross polar component is negligible. The cross polar filtering enhances the accuracy of the source reconstruction and accelerates the convergence.

Step 3) Calculate the PWS by applying the forward DFT to the field on the slots using (3).

Step 4) Replace the obtained reliable region of the PWS with the initial reliable region. During the iterative procedure, the initial reliable region is unchanged from the initial reliable region while the PWS outside the reliable region is updated until the convergence.

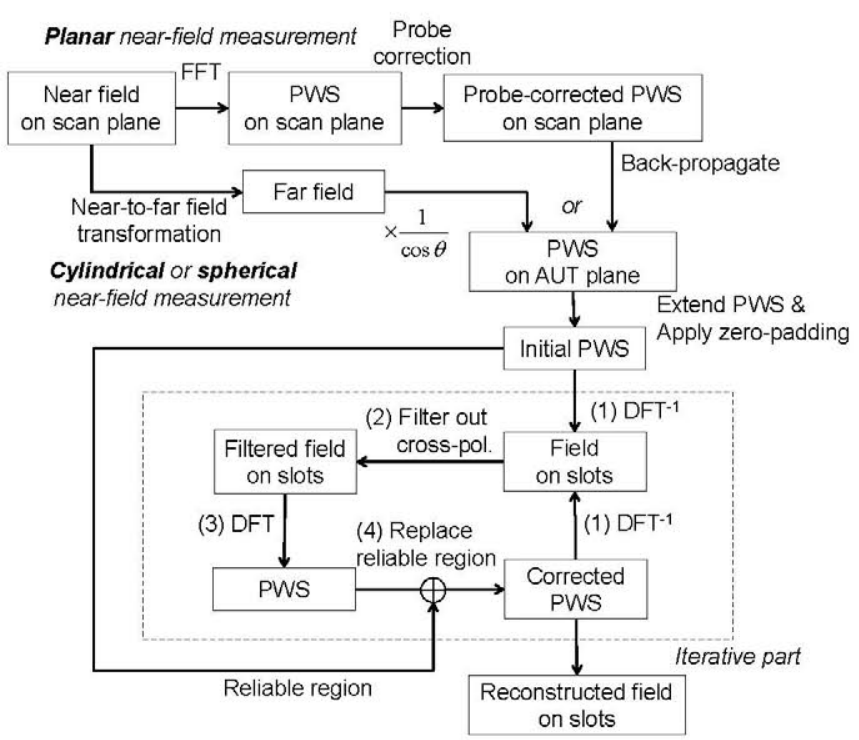

Fig. 3. Algorithm of the source reconstruction using the DFT.

\section{SOURCE RECONSTRUCTION FROM THE SIMULATED AND THE MEASURED DATA}

The source reconstruction technique has been applied to the simulated and the measured data of a K-band radial line slot antenna (RLSA) shown in Fig. 4. The RLSA consists of 1062 slots, and operates at $19.9 \mathrm{GHz}$. The initial PWS's for source reconstruction are computed from the simulated and the measured electric fields in the far-field region. The simulated far field is computed using the designed magnetic currents on the slots. As for the measurement, the RLSA is measured in a spherical near-field measurement system and the far field is obtained by the near-to-far field transformation [4]. In both simulation and measurement, the far field is sampled on a hemisphere with a $3^{\circ}$ step in $\theta$ for $0^{\circ} \leq \theta<90^{\circ}$, and a $3^{\circ}$ step in $\phi$ for $0^{\circ} \leq \phi<360^{\circ}$.

Fig. 5 shows the amplitudes and phases on the slots reconstructed after 50 iterations. The reconstructed amplitudes and phases in the graph are fitted to the designed ones so that error between the reconstructed and designed data is minimized. The simulated results almost overlap with the designed ones. Although the measured curve has ripples, the tendency is similar to that of the designed one.
Fig. 6 shows the convergence of the source reconstruction. The error $\varepsilon_{i}$ between the reconstructed and the designed fields at the $i$-th iteration, and the difference $\delta_{i}$ of the fields between the (i-1)-th and $i$-th iterations are plotted (in this case for both simulations and measurements). The error $\varepsilon_{i}$ and the difference $\delta_{i}$ are given by

$$
\begin{gathered}
\varepsilon_{i}=\frac{\sum_{n=1}^{N}\left|E^{(i)}\left(x_{n}, y_{n}\right)-E_{\text {design }}\left(x_{n}, y_{n}\right)\right|^{2}}{\sum_{n=1}^{N}\left|E_{\text {design }}\left(x_{n}, y_{n}\right)\right|^{2}} \\
\delta_{i}=\frac{\sum_{n=1}^{N}\left|E^{(i)}\left(x_{n}, y_{n}\right)-E^{(i-1)}\left(x_{n}, y_{n}\right)\right|^{2}}{\sum_{n=1}^{N}\left|E^{(i-1)}\left(x_{n}, y_{n}\right)\right|^{2}} \quad(i \geq 2)
\end{gathered}
$$

where $E^{(i)}\left(x_{n}, y_{n}\right)$ and $E_{\text {design }}\left(x_{n}, y_{n}\right)$ are the reconstructed and the designed fields at the position of the $n$-th slot, respectively. The error curve of the simulation shows the convergence just after the first 5 iterations, while difference curve shows the slower convergence.

The convergence of the method can be observed with the evolution of the results for the simulated and measured values with number of iterations. Fig. 7 shows the amplitude of the reconstructed field after 1 (direct application of the DFT), 3, 10, and 50 iterations compared with the designed values. The simulated results after 10 and 50 iterations are almost the same. This is because the error curve in Fig. 6 has reached the convergence after 10 iterations. However, as also can be seen in the Fig. 5 (a), the measurement result after 50 iterations has ripples in amplitude and seems to be deteriorated compared with the result after 10 iterations. This may be because the error of the reconstructed field is increasing due to the measurement error. In the case of measurements, the proper termination of the iteration using the convergence of the difference is required because we do not usually know the exact field on the slots as a reference.

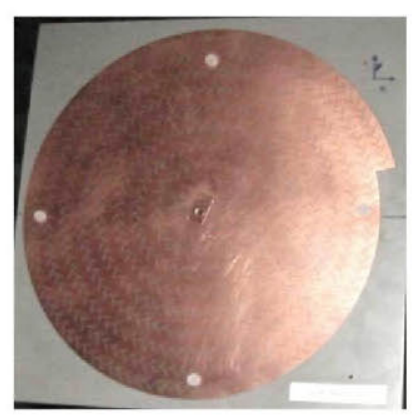

Fig. 4. Radial line slot array (RLSA) used for the source reconstruction. 


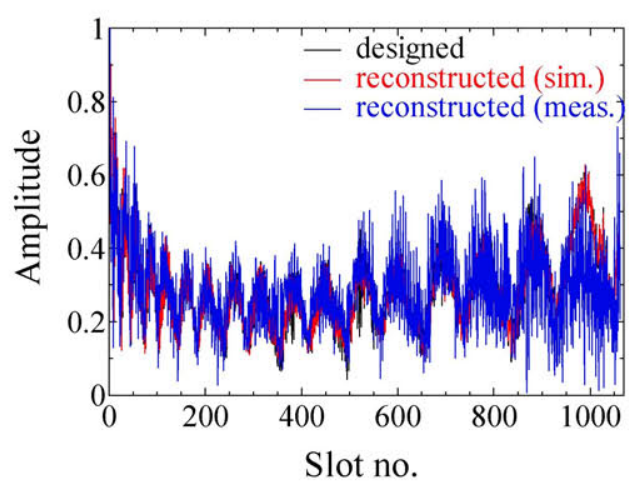

(a) Amplitude of the reconstructed field

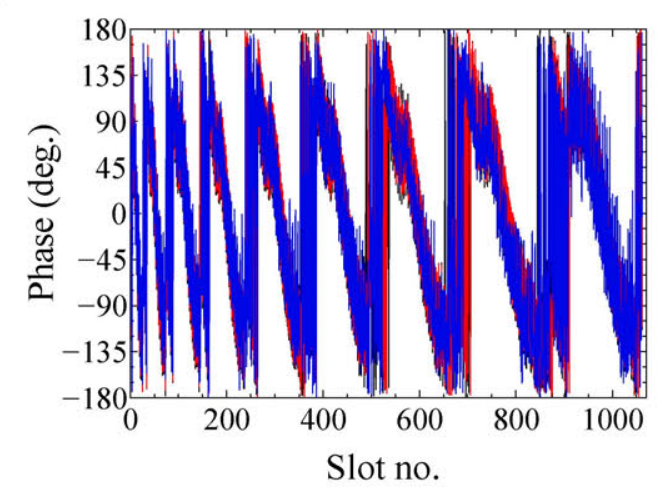

(b) Phase of the reconstructed field

Fig. 5. Amplitudes and phases of the field reconstructed from the simulated and the measured far-field patterns. The slots are numbered from the innermost slot.

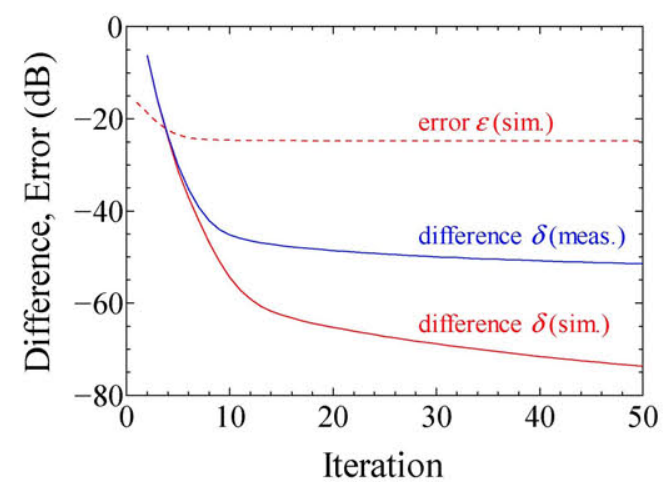

Fig. 6. Convergence of source reconstruction technique

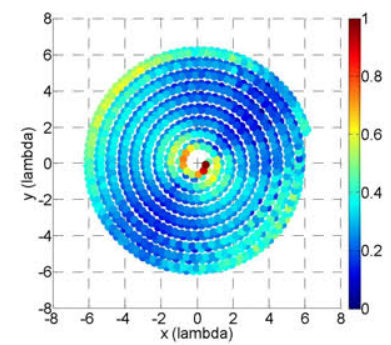

(a) designed

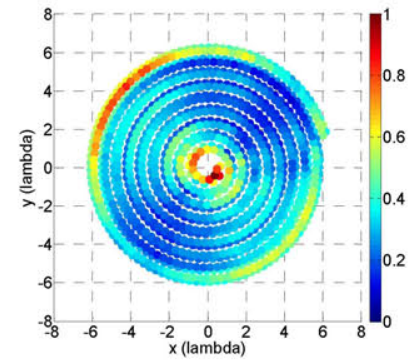

(b) after 1 iteration (simulated)

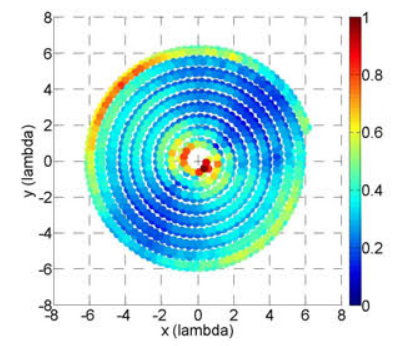

(d) after 3 iterations (simulated)

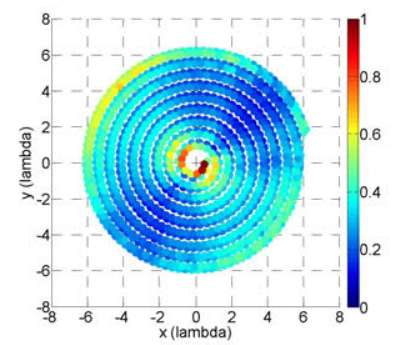

(f) after 10 iterations (simulated)

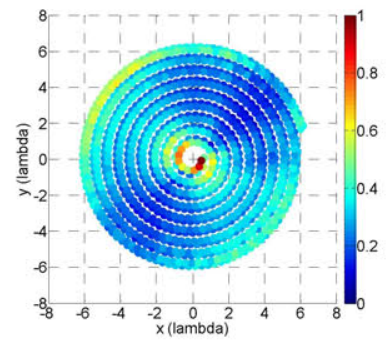

(h) after 50 iterations (simulated)

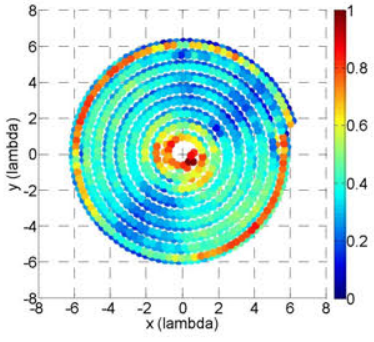

(c) after 1 iteration (measured)

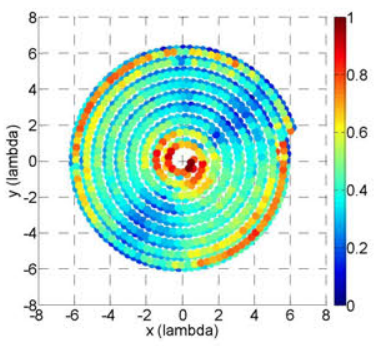

(e) after 3 iterations (measured)

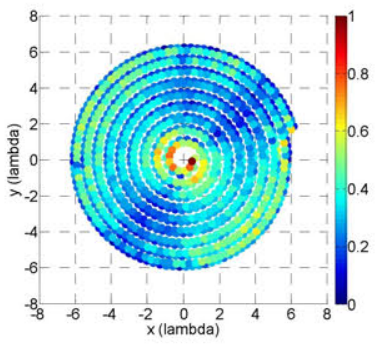

(g) after 10 iterations (measured)

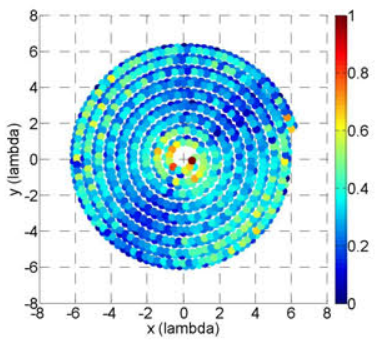

(i) after 50 iterations (measured)
Fig. 7. Evolution of the source reconstruction technique. The normalized amplitude of the reconstructed aperture electric field is plotted.

\section{CONCLUSION}

This authors have proposed a new source reconstruction technique applied for slot array antennas. The DFT has been adopted to the Gerchberg-Papoulis algorithm for the antenna measurements so as to compute the PWS from the electric field on the slots without interpolation. The inverse of the DFT is implemented using the pseudoinverse matrix. The proposed technique has been validated through the source reconstructions from the simulated and the measured far-field patterns. Although the proposed technique is based upon the 
assumption that the electric field is confined on the centers of the slots, we have confirmed good performance of the source reconstruction.

\section{Acknowledgment}

This work has been supported by European Union Erasmus Mundus BEAM program and by Tokyo Tech SERP Program.

\section{References}

[1] L. J. Foged, L. Scialacqua, F. Saccardi, J. L. Araque Quijano, G Vecchi, and M. Sabbadini,"Practical Application of the Equivalent Source Method as an Antenna Diagnostics Tool" Proceedings of the AMTA 2011 Symposium. October, 2011.

[2] J. J. H. Wang, "An examination of the theory and practices of planar near-field measurement," IEEE Trans. Antenns. and Propag., vol. 36, no. 6, pp. 746-753, June 1988.

[3] J. A. Straton, Electromagnetic Theory, ch. 6.7, New York: McGrowHill, 1941.
[4] A. D. Yaghjian, "An overview of near-field antenna measurements," IEEE Trans. Antennas and Propagat., vol. 34, no.1, pp. 30-45, Jan. 1986.

[5] A. C. Newell, "Error analysis techinique for planar near-field measurements," IEEE Trans. Antennas and Propagat., vol. 36, no. 6, pp. 754-768, June 1988.

[6] E. Martini, O. Breinbjerg, and S. Maci, "Reduction of truncation errors in planar near-field aperture antenna measurements using the Gerchberg-Papoulis algorithm," IEEE Trans. Antennas and Propag., vol. 56, no. 11, pp. 3485-3493, Nov. 2008.

[7] C. Cappellin, A. Frandsen, and O. Breinbjerg. "Application of the SWEto-PWE antenna diagnositics technique to an offset reflector antenna," IEEE Antennas Propag. Mag., vol. 50, no. 5, pp. 204-213, Oct. 2008.

[8] A. Papoulis, "A new algorithm in spectral analysis and band-limited extrapolation," IEEE Trans. Circuits Syst., vol. CAS-22, no. 9, pp. 735742, Sep. 1975.

[9] E. Martini, O. Breinbjerg, and S. Maci, "Reduction of truncation errors in planar near-field measruements using the method of alternating orhogonal projections," European Conference on Antennas and Propagation (EuCAP2006), Nice, Nov. 2006.

[10] A. B. Isreal and T. N. E. Greville, Generalized Inverses: Theory and Applications, ch. 1, Wiley, New-York, 1980. 